\title{
Treatment of open and comminuted mid-distal tibial fractures by bilateral external fixation combined with limited-internal fixation
}

\author{
Hongsuo Liang, Lin Li, Jingye Yang, Yongjun Du, Wuxun Peng \\ From the Department of Orthopaedic Surgery, Nanning Second People’s Hospital. Nanning, Guangxi, China
}

Open and comminuted mid-distal fractures often result from high-energy trauma, and a concomitant poor blood supply often leads to skin necrosis, infection, and bone union. To circumvent such complications, we used limited-reduction and bilateral-external fixators to treat open and comminuted mid-distal tibial fractures with compromised soft tissue. A retrospective series of 34 patients who had open and comminuted mid-distal tibial fractures and treated by bilateral-external fixators with limited-internal fixation were analyzed. Patients were followed for 10-25 months (mean: 12 months) post-treatment and osseous union was achieved in each case. The average union time was 16.3 weeks. Based on the JohnerWruhs criteria, the retrospective series consisted of 21 'excellent' cases, 8 'good' cases, 4 'fair' cases, and a 'poor' case. The total percentage of 'excellent' and 'good' cases of fracture recovery was $85.29 \%$. Bilateral-external and limited-internal fixators provided high bone union rate and excellent ankle-joint motion. Hence, it is an appropriate surgical approach for treating open and comminuted mid-distal tibial fractures with compromised soft tissue.

Keywords Tibial shaft fracture; External fixation; internal fixation.

\section{INTRODUCTION}

Open and comminuted fractures of the tibia and fibula are commonly caused by high-energy trauma, which has become a major challenge

Conflict of interest and source of funding: No funding was received in support of this study. The authors declare that they have no conflicts of interest. to orthopedic surgeons due to concomitant damage of accompanying soft tissues. Because of the thin soft-tissue coverage of the mid-distal tibia, open fractures-associated skin and muscle damages are common. Although open reduction and internal fixation provide efficacious fixation, these procedures may increase the risk of softtissue injury and bone-healing complications $(1,2)$, such as nonunion, delayed union, and infections. External fixation has advantages for the treatment of open and comminuted fractures. However, due to inadequate initial reduction and stable fixation or later reduction loss, external fixation may lead to some complications, including delayed union, malunion, and non-union (3-5). In the current study, we introduced a combined treatment of bilateralexternal and limited-internal fixators for open and

\footnotetext{
Hongsuo Liang $\mathrm{MD}^{1}$,

Lin $\mathrm{Li}^{1}$,

Jingye Yang ${ }^{1}$,

- Yongjun $\mathrm{Du}^{1}$,

- Wuxun Peng MD²

${ }^{1}$ Department of Orthopaedic Surgery, Nanning Second People's Hospital. Nanning, Guangxi, China.

${ }^{2}$ Department of Emergency Surgery, The Affiliated Hospital of Guizhou Medical University, Guiyang, Guizhou, China.

Correspondence : Wuxun Peng, Department of Emergency Surgery, The Affiliated Hospital of Guizhou Medical University,Guiyi Street,Guiyang, Guizhou, China. Phone: (86) 0851-86851119.

Email : pwx200228@aliyun.com

- 2021, Acta Orthopædica Belgica.
} 
comminuted mid-distal tibial fractures, with may be used as an alternative treatment option for fractures with compromised soft tissue.

\section{MATERIALS AND METHODS}

This study was approval by the Ethics Committee of the Nanning Second People's Hospital. From February 2014 to October 2016, 34 patients (21 males, 13 females) with open and displaced middistal tibial-shaft fractures (with or without a fibular fracture) were treated with bilateral-external and limited-internal fixators at our hospital. The inclusion criteria were as follows: 1) aged at least 18 years old; 2) having an open and comminuted fracture of the mid-distal tibia. Patients with associated proximal or distal intra-articular fractures of the tibia, fractures of the tibial plafond, fractures associated with vascular injury that required repair; or suspected pathologic fractures were excluded. The location and AO classification of the fractures were determined by radiographs. Patients were aged between 18 and 62 years with an average of 39.4 years. The mechanisms of injury included traffic accidents $(n=18)$, falls from heights $(n=9)$, and direct impact from heavy objects $(n=7)$. According to the Anderson-Gustilo classification, there were 11 Grade-II types, 14 Grade-IIIA types, and 9 GradeIIIB types. According to the AO classification, there were 4 B1 types, 7 B2 types, 5 B3 types, $13 \mathrm{C} 1$ types, and $5 \mathrm{C} 3$ types. Operations were performed at 2-hour to 7-day post-trauma. Informed consent was obtained from each patient.

Debridement of open fractures was routinely completed prior to external fixation. Broadspectrum antibiotics were started upon admission and were continued for 48-72 h. All open fractures were treated urgently in an operating room with thorough irrigation and debridement. The patient was under general or spinal anesthesia and placed on a radiolucent table in a supine position with a tourniquet placed on the proximal thigh of the fractured leg before operation. Operations were performed by the same group of surgeons. Necrotic tissues were debrided thoroughly during the operation. The wounds were stitched instantly without any soft-tissue defects. Patients were treated with vacuum-sealing drainage (VSD) if soft tissues were defected. Skin grafting or skin-flap transferring was then performed based on wound conditions. Open fractures of the tibula and fibula were cleaned and treated with a plaster cast or brace fixture. In patients with abdominal and/or head injuries, surgery was initiated once each patient's condition became stable. For simple and open multi-segment tibial fractures, soft-tissues injuries were debrided and sutured, and bone fractures were treated at the first stage with external fixation. To avoid insufficient anatomic reduction, especially for more complicated tibial-fracture patterns, a 4-6 cm-long incision was made anteriorly over the major fragments. The fractures were reduced under direct vision to achieve anatomic or near anatomic reduction. Screws and/or Kirschner wires were required for accessorial fixation of unstable fractures, such as oblique or spiral fractures. All anteroposterior or medial-lateral displacement were decreased. Two cross Kirschner wires were implanted vertical to the distal tibia via $\mathrm{C}$-arm fluoroscopy, specifically at $3-4 \mathrm{~cm}$ above the ankle joint. Two Kirschner wires were intersected with an angle of $60^{\circ}-80^{\circ}$ and placed trans-osseously distal to the fracture site, during which considerable caution was made to respect the security corridors and avoid adjacent neurovascular bundles. Three or four other Kirschner wires were implanted at least 6-8 $\mathrm{cm}$ apart from one another-vertical to the proximal tibia and parallel to the articular surface. The total diameter of these Kirschner wires were 3-4 mm. For some severe comminuted fractures, proximal Kirschner wires were instead used for cross-fixation to increase stability. Double external-fixator rods were assembled on each side of the tibia. The arms of the external fixator were kept 3-4 cm away from the skin of the leg. When adequate reduction was obtained, the clamp of the construct was locked and secured. If necessary, fibular fixation was performed percutaneously with pins, cannulated screws, or plates. The effect of ligamentotaxis on reduction was evaluated under C-arm fluoroscopy.

Clinical and radiological assessments were performed every month post-operation. All patients were encouraged to engage in early-function exercises. In general, patients were allowed to weight- 
bear as tolerated, starting at the fourth week after operation. Full weight-bearing activities were eventually allowed, according to the status of fracture healing. Patients were instructed to clean the site with medical alcohol every day and remove debris at the pin site with a sterile throat swab. Patients were then instructed to allow the pin site to dry and then to cover it with a sterile standard bandage. The fractures were considered healed when radiographs showed 3-4 cortical bridging or when the fracture lines disappeared, as well as no pain with weight-bearing. If there was any radiographic evidence of a bridging callus or the disappearance of the fracture lines, and no abnormal mobility or pain during walking, external fixator should be removed as soon as possible. All external fixators were removed under local anesthesia in outpatient department. Final results were evaluated based on radiological, clinical and subjective outcomes according to the standard criteria introduced by Johner and Wruhs (6).

\section{RESULTS}

All patients completed the clinical trial and followed for 10-25 months (average: 12 months). All wounds healed well without any complications. Two patients (Grade IIIB) with delayed flaps showed acceptable coverage. All fractures were ultimately united at follow-up, but delayed union was observed in two (2/34) patients, whose external fixations were adjusted to eliminate stress and their fractures eventually healed. The average fractureunion time was 16.3 weeks (range: 12-29 weeks). No external-fixation breakage or nonunion fracture occurred. Pin-tract infections were diagnosed based on wound discharge, swelling, and pain at the pin site and were verified by bacterial cultures. These infections were diagnosed in four cases and were effectively treated with oral antibiotics and localpin care. There was no case of deep infection or failure of fixation. According to the standard criteria for functional recovery by Johner and Wruhs, our retrospective series consisted of 21 'excellent' cases, eight 'good' cases, four 'fair' cases, and one 'poor' case. The total percentage of 'excellent' and 'good' cases of fracture recovery was $85.29 \%$. The
X-ray images of two typical cases before and after the surgery are shown in Figure 1 and 2.
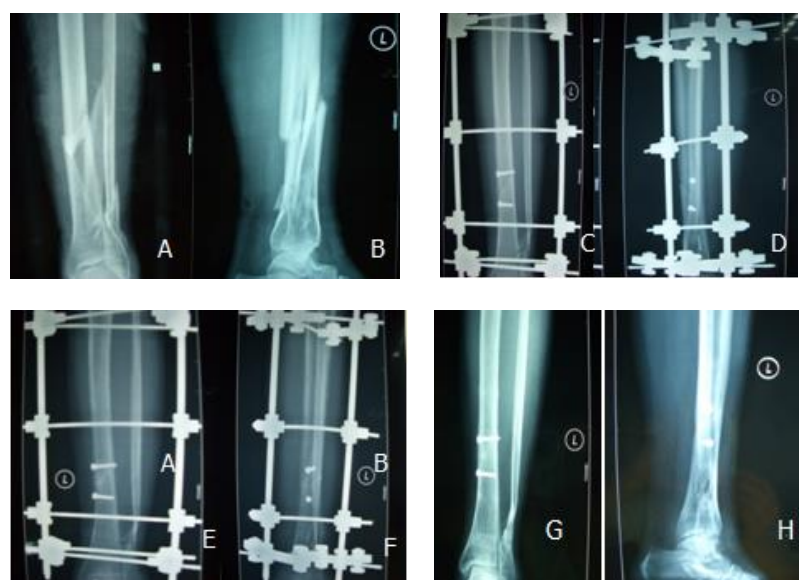

Figure 1. - A 38-year-old male with an AO-type/B3-type open left tibiofibular comminuted fracture was treated with a bilateral-external fixator combined with limited-internal fixation. Images at preoperative (A, B), early postoperative (C, $\mathrm{D})$, postoperative at 3 months $(\mathrm{E}, \mathrm{F})$, and postoperative after the removal of external fixation $(\mathrm{G}, \mathrm{H})$ are shown.
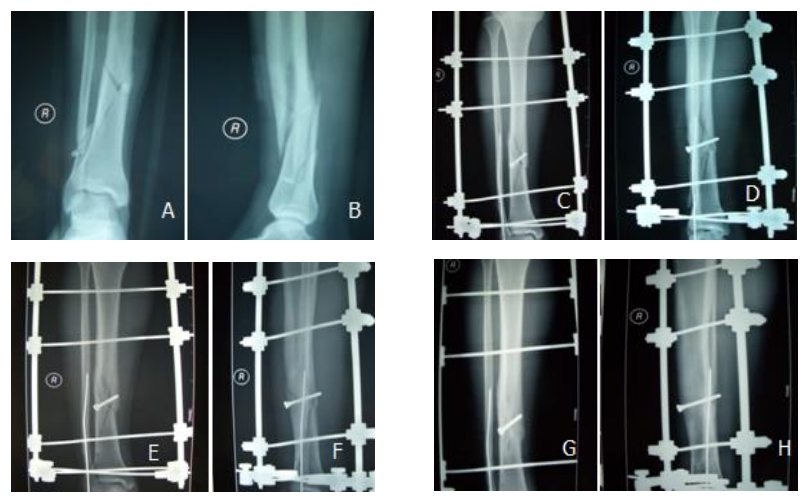

Figure 2. - A 29-year-old male with an AO-type/B2-type open right tibiofibular comminuted fracture was treated with a bilateral-external fixator combined with limited-internal fixation. Images at preoperative (A, B), early postoperative (C, $\mathrm{D})$, postoperative at 3 months $(\mathrm{E}, \mathrm{F})$, and postoperative at 5 months $(\mathrm{G}, \mathrm{H})$ are shown.

\section{DISCUSSION}

Open and comminuted mid-distal tibial fractures are a challenge for orthopedic surgeons. Fracture management depends on the status of the adjacent soft tissue and the degree of comminution. The mid-distal tibia is one of the locations prone to post- 
operative infection on account of its thin soft-tissue envelope as well as high incidence of open fractures. The slower healing of the distal tibia is possibly due to the lack of surrounding soft tissue, poor blood supply, and frequent recruitment for weight-bearing functions (7-9). Additionally, non-union or delayed union occurs frequently in patients with fractures of mid-distal segments of the tibia, especially in open and comminuted tibial fractures.

Intramedullary nailing, plate osteosynthesis, and external fixation are the most common fixation methods with their own advantages and indications $(10,11)$. It is more difficult to achieve anatomic alignment and adequate fixation using intramedullary-nail techniques in short distal-bone segment than those used in the midshaft due to the lack of diaphyseal fit. The incidence of infection, malunion, and nonunion is high, especially in patients with open fractures and multiple comorbidities (12). Conventional plating methods are unsuitable for open and comminuted tibial fractures because of poor soft tissue coverage and precarious blood supply (13). As to plate fixation, the subcutaneous location of the plates may also contribute to infection. Open reduction and plate/ screw fixation achieve anatomic reduction and stable ostheosynthesis for these fractures. However, soft-tissue complications, particularly wound dehiscence and infection, are major problems with the open-surgical technique, which can also induce the disruption of vascularity and lead to nonunion. Wound healing is also impacted by the insertion of a bulky plate into the dense soft-tissue envelope of the distal tibia. Sathiyakumar et al. (14) found that higher plate-osteosynthesis re-operation rate in open fractures versus closed fractures (46\% vs. 29\%), confirming that avoidance of plate osteosynthesis is a reference for open fractures. External fixators are the traditional fixation method recommended for contaminated open fractures and some closed fractures with severe soft-tissue injuries (15). Many studies used external fixation as a minimally invasive operation that often reduced the devitalization of soft tissue or bone $(4,16,17)$. This therapy is acceptable for tibial fractures along with severe soft-tissue injury. Because it is convenient to make the fixator dynamic to promote union with less secondary iatrogenic injury introduced (18). Unilateral external fixation is often used for high-energy fractures awaiting soft-tissue recovery since the application of this treatment is minimally invasive and is relatively easy and rapid. However, this approach is not suited well for definitive care because unilateral external fixation is relatively unstable at maintaining fracture alignment, especially over longer working lengths, and does not allow for early weight-bearing activity (19). Due to poor stability of external fixators, excessive interfragmentary movements may happen when taking weight-bearing functional exercises, so that the healing period would be prolonged and the union of the bone fragments would be delayed or even terminated. Alternatively, many researchers have used the Ilizarov circular-external fixator with smaller pins for such treatment. These frames do not cross the ankle joint. Besides, it is possible to stabilize the fracture soon after injury. Biomechanically, circular-external fixators are more rigid than unilateral-external fixators. However, the Ilizarov method still has obvious disadvantages with the frame installed, such as longer treatment time, long-term effects to walk, and more discomfort for bulky structure (20). Additionally, the inconvenience of the external fixator, the influence on exacerbating joint stiffness, and the frequent pin-tract infections are unacceptable complications for some patients $(21,22)$.

Bilateral-external fixation can make each arm participate in force simultaneously and reduce the stress associated with unilateral external fixation, providing maximal stability of the fracture ends. Bilateral-external fixation has a smaller volume and fewer pins compared with those of the Ilizarov device, which provides a smaller and lighter framework for patients. In our research of bilateralexternal fixation, pin-tract infection remained to be the most common complication with an incidence of $11.8 \%$, which was much lower than that for circular-external fixators in other reports $(4,23,24)$. The decrease of pin-tract infection in our present study was likely due to the rigid fixation of our method and the relatively earlier removal of the external fixator. Additionally, according to the status of external fixation and fracture healing, bilateralexternal fixation can be adjusted to enhanced 
compression or dynamic compression in order to further promote fracture healing. In this study, the two cases with delayed fracture union eventually healed by adjusting the degree of tightness of the clips of the bilateral-external fixator.

There are often large free-bone fragments in open and comminuted tibial fractures at the middistal portion of the tibia. Because of severe softtissue injury, manual reduction is not easy during operation and it is difficult to achieve anatomic reduction. Repeated manual reduction can aggravate the secondary injury of the accompanying leg tissue. It is difficult to use external fixation alone to treat open and comminuted tibial fractures, such as long oblique and spiral fractures. For such unstable open fractures, a single fixator cannot obtain effective reduction and stable fixation effect (25). The application of external fixators can reduce the occurrence of soft-tissue necrosis and deep infection. However, when they are used as a definitive fixation device, high incidence of malunion would happen even with circular-external fixators due to inadequate rigidity (26). Therefore, it is necessary for open reductions to obverse directly and for fracture fragments to take internal fixation. Open reduction and internal fixation can achieve anatomic reduction for comminuted fractures (30). The combination of external fixation and minimal internal fixation involves both mechanical and biological treatment for fracture. Most reduction is achieved by ligamentotaxis, so that extensive surgical dissection can be avoided (8). The combined application of limited open-reduction and external fixators can make the fracture-fixation stronger, which can enable earlier onset of postsurgery exercise and promote healing of the fracture and rehabilitation of limb function (27). For these reasons, the bilateral-external fixator was removed with a mean time of 16.3 weeks (range: 12-29 weeks). which was relatively less time than other reports that only used an external fixator (28,29). Additionally, efficacious fracture reduction contributes to improving patient satisfaction and decreasing doctor-patient disputes. Open reduction can also relieve pressure from the lower legs, which helps to circumvent the occurrence of osteofascialcompartment syndrome. The combination of ex- ternal fixation with minimal-internal fixation can promote fracture healing mechanically and biologically. Thus, we conclude that minimal-internal fixation combined with external fixation yields efficacious initial reduction of fractures. With this approach, extensive surgical dissection is avoided and reduction is achieved largely through ligamentotaxis.

\section{CONCLUSIONS}

This preliminary study indicated that a bilateralexternal and limited-internal fixation system, as a novel external- and intra-fixation technique, respectively, had minimal invasiveness, but provided sufficient stability for the selective treatment of open and comminuted mid-distal tibial fractures. For cases that are not suitable for the use of external fixators, plates, or other routine methods, this combinational technique may be applied. These cases will mostly consist of open/comminuted and/ or unstable fractures with accompanied by softtissue complications. Our method allows for early definitive treatment with a low complication rate and efficacious clinical outcome.

\section{Acknowledgments}

We thank LetPub (www.letpub.com) for its linguistic assistance during the preparation of this manuscript.

\section{REFERENCES}

1. Song X, Huang X, Yakufu M, Yan B, Feng C. Minimally invasive plate osteosynthesis or conventional intramedullary nailing for distal tibial fractures: A cohort study protocol. Medicine. 2020; 99(33): e21779.

2. Van de Wall BJM, Theus C, Link BC, van Veelen N, van de Leeuwen RJH, Ganzert $\mathrm{C}$, et al. Absolute or relative stability in plate fixation for simple humeral shaft fractures. Injury. 2019; 50(11): 1986-91.

3. Ronga M, Longo UG, Maffulli N. Minimally invasive locked plating of distal tibia fractures is safe and effective. Clin Orthop Relat Res. 2010; 468(4): 975-82.

4. Anglen JO. Early outcome of hybrid external fixation for fracture of the distal tibia. J Orthop Trauma. 1999; 13(2): 92-7.

5. Galal S. Minimally invasive plate osteosynthesis has equal safety to reamed intramedullary nails in treating GustiloAnderson type I, II and III-A open tibial shaft fractures. Injury. 2018; 49(4): 866-70. 
6. Dall'Oca C, Christodoulidis A, Bortolazzi R, Bartolozzi P, Lavini F. Treatment of 103 displaced tibial diaphyseal fractures with a radiolucent unilateral external fixator. Arch Orthop Trauma Surg. 2010; 130(11): 1377-82.

7. Li Y, Jiang X, Guo Q, Zhu L, Ye T, Chen A. Treatment of distal tibial shaft fractures by three different surgical methods: a randomized, prospective study. Int Orthop. 2014; 38(6): 1261-7.

8. Osman W, Alaya Z, Kaziz H, Hassini L, Braiki M, Naouar $\mathbf{N}$, et al. Treatment of high-energy pilon fractures using the ILIZAROV treatment. Pan Afr Med J. 2017; 27(1): 199.

9. Ramos T, Karlsson J, Eriksson BI, Nistor L. Treatment of distal tibial fractures with the Ilizarov external fixator-a prospective observational study in 39 consecutive patients. BMC Musculoskelet Disord. 2013; 14(1): 1-12.

10. Li H, Yu D, Wu S, Zhang Y, Ma L. Multiple Comparisons of the Efficacy and Safety for Seven Treatments in Tibia Shaft Fracture Patients. Front Pharmacol. 2019; 10: 197.

11. Tarkin IS, Siska PA, Zelle BA. Soft tissue and biomechanical challenges encountered with the management of distal tibia nonunions. Orthop Clin North Am. 2010; 41(1): 119-26.

12. Avilucea FR, Sathiyakumar V, Greenberg SE, Ghiam M, Thakore RV, Francois E, et al. Open distal tibial shaft fractures: a retrospective comparison of medial plate versus nail fixation. Eur J Trauma Emerg Surg. 2016; 42(1): 1016.

13. Bell A, Templeman D, Weinlein JC. Nonunion of the femur and tibia: an update. Orthop Clin. 2016; 47(2): 36575.

14. Cabrolier J, Molina M. Is intramedullary nailing superior to plating in patients with extraarticular fracture of the distal tibia? Medwave. 2015; 15(Suppl 3): e6306.

15. Fadel M, Ahmed MA, Al-Dars AM, Maabed MA, Shawki H. Ilizarov external fixation versus plate osteosynthesis in the management of extra-articular fractures of the distal tibia. Int Orthop. 2015; 39(3): 513-9.

16. Pairon P, Ossendorf C, Kuhn S, Hofmann A, Rommens PM. Intramedullary nailing after external fixation of the femur and tibia: a review of advantages and limits. Eur $J$ Trauma Emerg Surg. 2015; 41(1): 25-38.

17. Polat A, Kose O, Canbora K, Yanık S, Guler F. Intramedullary nailing versus minimally invasive plate osteosynthesis for distal extra-articular tibial fractures: a prospective randomized clinical trial. J Orthop Sci. 2015; 20(4): 695-701.
18. Sathiyakumar V, Thakore RV, Ihejirika RC, Obremskey WT, Sethi MK. Distal tibia fractures and medial plating: factors influencing re-operation. Int Orthop. 2014; 38(7): 1483-8.

19. Ang BFH, Chen JY, Yew AKS, Chua SK, Chou SM, Chia SL, et al. Externalised locking compression plate as an alternative to the unilateral external fixator: a biomechanical comparative study of axial and torsional stiffness. Bone Joint Res. 2017; 6(4): 216-23.

20. Barbieri R, Schenk R, Koval K, Aurori K, Aurori B. Hybrid external fixation in the treatment of tibial plafond fractures. Clin Orthop Relat Res. 1996; 332: 16-22.

21. Bone L, Stegemann P, McNamara K, Seibel R. External fixation of severely comminuted and open tibial pilon fractures. Clin Orthop Relat Res. 1993(292): 101-7.

22. Koulouvaris P, Stafylas K, Mitsionis G, Vekris M, Mavrodontidis A, Xenakis T. Long-term results of various therapy concepts in severe pilon fractures. Arch Orthop Trauma Surg. 2007; 127(5): 313-20.

23. Quinnan SM. Definitive management of distal tibia and simple plafond fractures with circular external fixation. $J$ Orthop Trauma. 2016; 30: S26-S32.

24. Zhou Y, Wang Y, Liu L, Zhou Z, Cao X. Locking compression plate as an external fixator in the treatment of closed distal tibial fractures. Int Orthop. 2015; 39(11): 2227-37.

25. El-Rosasy MA, El-Sallakh SA. Distal tibial hypertrophic nonunion with deformity: treatment by fixator-assisted acute deformity correction and LCP fixation. Strategies Trauma Limb Reconstr. 2013; 8(1): 31-5.

26. Sanders DW, Galpin RD, Hosseini M, MacLeod MD. Morbidity resulting from the treatment of tibial nonunion with the Ilizarov frame. Can J Surg. 2002; 45(3): 196-200.

27. Sigurdsen U, Reikeras O, Utvag SE. Conversion of external fixation to definitive intramedullary nailing in experimental tibial fractures. J Invest Surg. 2010; 23(3): $142-8$.

28. Ramos T, Eriksson BI, Karlsson J, Nistor L. Ilizarov external fixation or locked intramedullary nailing in diaphyseal tibial fractures: a randomized, prospective study of 58 consecutive patients. Arch Orthop Trauma Surg. 2014; 134(6): 793-802.

29. Meena UK, Bansal MC, Behera P, Upadhyay R, Gothwal GC. Evaluation of functional outcome of pilon fractures managed with limited internal fixation and external fixation: A prospective clinical study. J Clin Orthop Trauma. 2017; 8: S16-S20. 